\title{
OPTIMAL REARRANGEMENT INVARIANT SOBOLEV EMBEDDINGS IN MIXED NORM SPACES
}

\author{
NADIA CLAVERO AND JAVIER SORIA
}

\begin{abstract}
We improve the Sobolev-type embeddings due to Gagliardo [20] and Nirenberg [28] in the setting of rearrangement invariant (r.i.) spaces. In particular we concentrate on seeking the optimal domains and the optimal ranges for these embeddings between r.i. spaces and mixed norm spaces. As a consequence, we prove that the classical estimate for the standard Sobolev space $W^{1} L^{p}$ by Poornima [31, O'Neil 29] and Peetre [30] $(1 \leq p<n)$, and by Hansson [21, Brezis and Wainger [12] and Maz'ya [26] $(p=n)$ can be further strengthened by considering mixed norms on the target spaces.
\end{abstract}

\section{INTRODUCTION}

Let $n \in \mathbb{N}$, with $n \geq 2$, and let $I \subset \mathbb{R}$ be an interval. The Sobolev space $W^{1} L^{p}\left(I^{n}\right)$, $1 \leq p \leq \infty$, consists of all functions in $L^{p}\left(I^{n}\right)$ whose first-order distributional derivatives also belong to $L^{p}\left(I^{n}\right)$. The classical Sobolev embedding theorem claims that

$$
W^{1} L^{p}\left(I^{n}\right) \hookrightarrow L^{p n /(n-p)}\left(I^{n}\right), \quad 1 \leq p<n .
$$

Sobolev [32] proved this embedding for $p>1$, but his method, based on integral representations, did not work when $p=1$. That case was settled affirmatively by Gagliardo [20] and Nirenberg [28], who first observed that

$$
W^{1} L^{1}\left(I^{n}\right) \hookrightarrow \mathcal{R}\left(L^{1}, L^{\infty}\right),
$$

(see Definition 3.2) and then, using an iterated form of Hölder's inequality, completed the proof; i.e.,

$$
W^{1} L^{1}\left(I^{n}\right) \hookrightarrow \mathcal{R}\left(L^{1}, L^{\infty}\right) \hookrightarrow L^{n^{\prime}}\left(I^{n}\right),
$$

where $n^{\prime}$ denotes the conjugate exponent of $n$, i.e., $1 / n+1 / n^{\prime}=1$.

Later, a new approach based on properties of mixed norm spaces was introduced by Fournier [19] and was subsequently developed, via different methods, by various authors, including Blei and Fournier [8, Milman [27], Algervik and Kolyada [2] and Kolyada [24, 25]. To be more precise, the central part of Fournier's work was to study embeddings between mixed norm spaces and Lorentz spaces $L^{p, q}$ (see Sections 2 for further details on Lorentz spaces). Specifically, he proved that

$$
\mathcal{R}\left(L^{1}, L^{\infty}\right) \hookrightarrow L^{n^{\prime}, 1}\left(I^{n}\right),
$$

2010 Mathematics Subject Classification. 28A35, 46E30, 46E35.

Key words and phrases. Sobolev embeddings; rearrangement-invariant spaces; Hardy operator; optimal range; optimal domain.

Both authors have been partially supported by the grants MTM2013-40985-P (Spanish Government) and 2014SGR289 (Catalan Autonomous Government). 
and then taking into account (2), he obtained the following improvement of (11):

$$
W^{1} L^{1}\left(I^{n}\right) \hookrightarrow L^{n^{\prime}, 1}\left(I^{n}\right) .
$$

The embedding (3) is due to Poornima [31, and it can be also traced in the works of O'Neil [29] and Peetre [30].

A thorough study of mixed norm spaces has been recently considered in [16]. In particular, extending the mixed norm estimates due to Fournier [19] to more general r.i. spaces, we have obtained a description of the largest mixed norm space of the form $\mathcal{R}\left(X, L^{\infty}\right)$ that is continuously embedded into a fixed r.i. space.

In recent years, extensions of (11) for more general rearrangement invariant (r.i.) spaces have been extensively studied by various authors, including Edmunds, Kerman and Pick [18, Kerman and Pick [23] and Cianchi [15]. To be more specific, Kerman and Pick [23] were interested on seeking necessary and sufficient conditions for the following embeddings involving r.i. spaces to hold:

$$
W^{1} Z\left(I^{n}\right) \hookrightarrow X^{\mathrm{op}}\left(I^{n}\right) .
$$

This characterization was then exploited to study the optimal domain-range problems for the embedding (4), within the class of r.i. spaces.

All these works provide us a strong motivation to consider (2) for more general r.i. spaces, as well as to describe the optimal domain and the optimal range for this embedding between r.i. spaces and mixed norm spaces.

The paper is organized as follows. In Sections 2 and 3, we present some basic properties of r.i. spaces and mixed norm spaces we shall need for our work.

Section 4 is devoted to study the Sobolev embedding of the form

$$
W^{1} Z\left(I^{n}\right) \hookrightarrow \mathcal{R}\left(X, L^{\infty}\right),
$$

extending the classical estimate (2). Following the ideas of [23], we establish the equivalence between (5) and the boundedness of a Hardy type operator (see Theorem 4.2). This relation will be a key tool to determine the optimal domain and the optimal range for (5) between r.i. spaces and mixed norm spaces.

After this discussion, our analysis focuses on giving explicit constructions of such optimal spaces. In particular, Theorem 4.4 provides a characterization of the smallest space of the form $\mathcal{R}\left(X, L^{\infty}\right)$ in (5), once the r.i. space $Z\left(I^{n}\right)$ is given. Finally, for a fixed mixed norm space $\mathcal{R}\left(X, L^{\infty}\right)$, Theorem 4.9 describes the largest r.i. space $Z$ for which (5) holds.

All these results are then employed to establish classical Sobolev embeddings in the context of mixed norm spaces. Thus, for instance, we recover (2) and, as a new contribution, we show that $\mathcal{R}\left(L^{1}, L^{\infty}\right)$ is the smallest mixed norm space of the form $\mathcal{R}\left(X, L^{\infty}\right)$ satisfying (2) .

As we have mentioned before, the optimal range problem for the Sobolev embedding was studied in [23] within the class of r.i. spaces. In particular, for a fixed r.i. domain space $Z\left(I^{n}\right)$ they determined the smallest r.i. space, namely $X^{\mathrm{op}}\left(I^{n}\right)$, satisfying (44). Motivated by this problem, in Section 5 we compare the optimal r.i. range space with the optimal mixed norm space, and we prove in Theorem 5.3 that the following chain of embeddings holds:

$$
W^{1} Z\left(I^{n}\right) \hookrightarrow \mathcal{R}\left(X, L^{\infty}\right) \hookrightarrow X^{\mathrm{op}}\left(I^{n}\right),
$$


with $\mathcal{R}\left(X, L^{\infty}\right)$ the mixed norm space constructed in Theorem 4.4. Consequently, it turns out that it is still possible to further improve the classical Sobolev embeddings by means of mixed norm spaces.

Some remarks about the notation: The measure of the unit ball in $\mathbb{R}^{n}$ will be represented by $\omega_{n}$. As usual, we use the symbol $A \lesssim B$ to indicate that there exists a universal positive constant $C$, independent of all important parameters, such that $A \leq C B$. The equivalence $A \approx B$ means that $A \lesssim B$ and $B \lesssim A$. Finally, the arrow $\hookrightarrow$ stands for a continuous embedding.

\section{Preliminaries}

We collect in this section some basic notations and results that will be useful in what follows.

Let $n \in \mathbb{N}$, with $n \geq 1$ and let $I \subset \mathbb{R}$ be an interval having Lebesgue measure $|I|=1$. We write $\mathcal{M}\left(I^{n}\right)$ for the set of all real-valued measurable functions on $I^{n}$ and $\mathcal{M}_{+}\left(I^{n}\right)$ for the non-negative ones.

Given $f \in \mathcal{M}\left(I^{n}\right)$, its distribution function $\lambda_{f}$ is defined by

$$
\lambda_{f}(t)=\left|\left\{x \in I^{n}:|f(x)|>t\right\}\right|, \quad t \geq 0,
$$

and the decreasing rearrangement $f^{*}$ of $f$ is defined as

$$
f^{*}(t)=\inf \left\{s \geq 0: \lambda_{f}(s) \leq t\right\}, \quad t \geq 0 .
$$

It is easily seen that if $h$ is a nonnegative and decreasing functions on $(0,1)$ then

$$
g(x)=h\left(\omega_{n}|x|^{n}\right) \text {, a.e. } x \Longrightarrow g^{*}=h^{*} .
$$

As usual, we shall use the notation $f^{* *}(t)=t^{-1} \int_{0}^{t} f^{*}(s) d s$. A basic property of rearrangements is the Hardy-Littlewood inequality (cf. e.g. [6, Theorem II.2.2]), which says:

$$
\int_{I^{n}}|f(x) g(x)| d x \leq \int_{0}^{1} f^{*}(t) g^{*}(t) d t, \quad f, g \in \mathcal{M}\left(I^{n}\right) .
$$

A Banach function norm $\rho$ is a mapping $\rho: \mathcal{M}_{+}\left(I^{n}\right) \rightarrow[0, \infty]$ such that the following properties hold:

(A1) $\rho(f)=0 \Leftrightarrow f=0$ a.e., $\rho(f+g) \leq \rho(f)+\rho(g), \rho(\alpha f)=\alpha \rho(f)$, for $\alpha \geq 0$;

(A2) if $0 \leq g \leq f$ a.e., then $\rho(g) \leq \rho(g)$;

(A3) if $0 \leq f_{j} \uparrow f$ a.e., then $\rho\left(f_{j}\right) \uparrow \rho(f)$;

(A4) $\rho\left(\chi_{I^{n}}\right)<\infty$;

(A5) $\int_{I^{n}}|f(x)| d x \lesssim \rho(f)$.

By means of $\rho$, a Banach function space $X\left(I^{n}\right)$ can be defined:

$$
X\left(I^{n}\right)=\left\{f \in \mathcal{M}\left(I^{n}\right): \rho(|f|)<\infty\right\} .
$$

For each $f \in X\left(I^{n}\right)$, we define $\|f\|_{X\left(I^{n}\right)}=\rho(|f|)$.

A Banach function norm is rearrangement invariant if $\|f\|_{X\left(I^{n}\right)}=\|g\|_{X\left(I^{n}\right)}$, for every pair of functions $f, g$ which are equimeasurable, that is, $\lambda_{f}=\lambda_{g}$. This means that the norm of a function $f$ in $X\left(I^{n}\right)$ depends only on its distribution function. In this case, we say that the Banach function space $X\left(I^{n}\right)$ is rearrangement invariant (briefly an r.i. space). 
The Lebesgue spaces $L^{p}\left(I^{n}\right)$, with $1 \leq p \leq \infty$, endowed with the standard norm, are the simplest example of r.i. spaces. We shall also work with the Lorentz spaces, defined either for $p=q=1$ or $p=q=\infty$, or $1<p<\infty$ and $1 \leq q \leq \infty$ as

$$
L^{p, q}\left(I^{n}\right)=\left\{f \in \mathcal{M}\left(I^{n}\right):\|f\|_{L^{p, q\left(I^{n}\right)}}<\infty\right\},
$$

where

$$
\|f\|_{L^{p, q}\left(I^{n}\right)}=\left\|t^{1 / p-1 / q} f^{*}(t)\right\|_{L^{q}(0,1)},
$$

and, more generally, with the Lorentz-Zygmund spaces, defined for $1 \leq p, q \leq \infty$ and $\alpha \in \mathbb{R}$ as

$$
L^{p, q ; \alpha}\left(I^{n}\right)=\left\{f \in \mathcal{M}\left(I^{n}\right):\|f\|_{L^{p, q ; \alpha}\left(I^{n}\right)}<\infty\right\}
$$

where

$$
\|f\|_{L^{p, q ; \alpha}\left(I^{n}\right)}=\left\|t^{1 / p-1 / q}[1+\log (1 / t)]^{\alpha} f^{*}(t)\right\|_{L^{q}(0,1)} .
$$

Observe that $L^{p, p}\left(I^{n}\right)=L^{p}\left(I^{n}\right)$ and $L^{p, q ; 0}\left(I^{n}\right)=L^{p, q}\left(I^{n}\right)$. Let us also mention, for the sake of completeness, that the quantities $\|\cdot\|_{L^{p, q}\left(I^{n}\right)}$ and $\|\cdot\|_{L^{p, q ; \alpha}\left(I^{n}\right)}$ are in general only quasi-norms, since they may fail to satisfy the triangle inequality. In most cases, they can be turned into equivalent norms replacing $f^{*}$ by $f^{* *}$ in corresponding definitions. However, when the weights $t^{1 / p-1 / q}$ or $t^{1 / p-1 / q}[1+\log (1 / t)]^{\alpha}$ are nonincreasing (and hence, in all cases we are going to consider), then $\|\cdot\|_{L^{p, q}\left(I^{n}\right)}$ and $\|\cdot\|_{L^{p, q ; \alpha}\left(I^{n}\right)}$ are norms.

Given an r.i. space $X\left(I^{n}\right)$, the set

$$
X^{\prime}\left(I^{n}\right)=\left\{f \in \mathcal{M}\left(I^{n}\right): \int_{I^{n}}|f(x) g(x)| d x<\infty, \text { for any } g \in X\left(I^{n}\right)\right\},
$$

equipped with the norm

$$
\|f\|_{X^{\prime}\left(I^{n}\right)}=\sup _{\|g\|_{X\left(I^{n}\right)} \leq 1} \int_{I^{n}}|f(x) g(x)| d x,
$$

is called the associate space of $X\left(I^{n}\right)$. It turns out that $X^{\prime}\left(I^{n}\right)$ is again an r.i. space [6, Theorem I.2.2]. The fundamental function of an r.i. space $X\left(I^{n}\right)$ is given by

$$
\varphi_{X}(t)=\left\|\chi_{E}\right\|_{X\left(I^{n}\right)},
$$

where $|E|=t$ and $\chi_{E}$ denotes the characteristic function of the set $E \subset I^{n}$.

A basic tool for working with r.i. spaces is the Hardy-Littlewood-Pólya Principle [6, Theorem II.2.2], which asserts that if $f \in X\left(I^{n}\right)$ and

$$
\int_{0}^{t} g^{*}(s) d s \leq \int_{0}^{t} f^{*}(s) d s, \quad 0<t<1
$$

then $g \in X\left(I^{n}\right)$ and $\|g\|_{X\left(I^{n}\right)} \leq\|f\|_{X\left(I^{n}\right)}$.

For later purposes, let us recall the Luxemburg representation theorem [6, Theorem II.4.10]. It says that given an r.i. space $X\left(I^{n}\right)$, there exists another r.i. space $\bar{X}(0,1)$ such that

$$
f \in X\left(I^{n}\right) \Longleftrightarrow f^{*} \in \bar{X}(0,1),
$$

and in this case $\|f\|_{X\left(I^{n}\right)}=\left\|f^{*}\right\|_{\bar{X}(0,1)}$. 
Next, we recall the definition of the Boyd indices of an r.i. space. First we introduce the dilation operator: if $f \in \mathcal{M}(0,1)$ and $t>0$,

$$
E_{t} f(s)= \begin{cases}f(s / t), & \text { if } 0 \leq s \leq \min (1, t) \\ 0, & \text { otherwise }\end{cases}
$$

It is well-known that the operator $E_{t}$ is bounded on $\bar{X}(0,1)$, for every r.i. space $X\left(I^{n}\right)$ and for every $t>0$ (see e.g. [6, Proposition III.5.11]).

By means of the norm of $E_{t}$ on $\bar{X}(0,1)$, denoted as $h_{X}(t)$, we define the lower and upper Boyd indices of $X\left(I^{n}\right)$ as

$$
\underline{\alpha}_{X}=\sup _{0<t<1} \frac{\log h_{X}(t)}{\log (t)} \text { and } \bar{\alpha}_{X}=\inf _{1<t<\infty} \frac{\log h_{X}(t)}{\log (t)} .
$$

It is easy to see that $0 \leq \underline{\alpha}_{X} \leq \bar{\alpha}_{X} \leq 1$. For instance, if $X=L^{p, q}$, then $h_{X}(t)=t^{1 / p}$ and thus $\underline{\alpha}_{X}=\bar{\alpha}_{X}=1 / p$. Furthermore, for later purposes, let us emphasize that $\bar{\alpha}_{L^{\infty, p ;-1}}=0, \quad 1<p<\infty$ (for more details see [6, 5]).

Let us next recall some special results from Interpolation Theory, which we shall need in what follows (for further information on this topic see [6, 7]).

Given a pair of compatible Banach spaces $\left(X_{0}, X_{1}\right)$ (compatible in the sense that they are continuously embedded into a common Hausdorff topological vector space), their $K$-functional is defined, for each $f \in X_{0}+X_{1}$, by

$$
K\left(f, t ; X_{0}, X_{1}\right):=\inf _{f=f_{0}+f_{1}}\left(\left\|f_{0}\right\|_{X_{0}}+t\left\|f_{1}\right\|_{X_{1}}\right), \quad t>0 .
$$

The fundamental result concerning the $K$-functional is the following [6, Theorem V.1.11]:

Theorem 2.1. Let $\left(X_{0}, X_{1}\right)$ and $\left(Y_{0}, Y_{1}\right)$ be two compatible pairs of Banach spaces and let $T$ be a sublinear operator satisfying

$$
T: X_{0} \rightarrow Y_{0} \text {, and } T: X_{1} \rightarrow Y_{1} \text {. }
$$

Then, there exists a constant $C>0$ (depending only on the norms of $T$ between $X_{0}$ and $Y_{0}$ and between $X_{1}$ and $Y_{1}$ ) such that

$$
K\left(T f, t ; Y_{0}, Y_{1}\right) \leq C K\left(C f, t ; X_{0}, X_{1}\right), \text { for every } f \in X_{0}+X_{1} \text { and } t>0 .
$$

The $K$-functional for pairs of Lorentz spaces $L^{p, q}\left(I^{n}\right)$ is given, up to equivalence, by the following result.

Theorem 2.2. (Holmstedt's formulas [22, Theorem 4.2]) Let $p_{0}=q_{0}=1$ or $1<$ $p_{0}<\infty$ and $1 \leq q_{0}<\infty$. Let $1 / \alpha=1 / p_{0}-1 / p_{1}$. Then,

$$
K\left(f, t ; L^{p_{0}, q_{0}}\left(I^{n}\right), L^{\infty}\left(I^{n}\right)\right) \approx\left(\int_{0}^{t^{p_{0}}}\left[s^{1 / p_{0}-1 / q_{0}} f^{*}(s)\right] d s\right)^{1 / q_{0}}, \text { for } t>0 .
$$

The first-order Sobolev space built upon an r.i. space $Z\left(I^{n}\right)$ is defined as

$$
W^{1} Z\left(I^{n}\right)=\left\{u \in Z\left(I^{n}\right):|\nabla u| \in Z\left(I^{n}\right)\right\},
$$

endowed with the norm

$$
\|u\|_{W^{1} Z\left(I^{n}\right)}=\|u\|_{Z\left(I^{n}\right)}+\|\mid \nabla u\|_{Z\left(I^{n}\right)} .
$$


Here, $\nabla u$ stands for the gradient of $u$ and $|\nabla u|=\left(\sum_{i=1}^{n} u_{x_{i}}^{2}\right)^{1 / 2}$. Observe that if $\left|D^{1} u\right|$ denotes the Euclidean length of $(u, \nabla u)$ as an element of $\mathbb{R}^{n+1}$, then

$$
\|u\|_{W^{1} Z\left(I^{n}\right)} \approx\left\|\left|D^{1} u\right|\right\|_{Z\left(I^{n}\right)} .
$$

Concerning the $K$-functional for a couple of Sobolev spaces, we mention the work of DeVore and Scherer [17], who proved that, for every $u \in W^{1} L^{1}\left(I^{n}\right)$,

$$
K\left(u, t ; W^{1} L^{1}\left(I^{n}\right), W^{1} L^{\infty}\left(I^{n}\right)\right) \approx \int_{0}^{t}\left|D^{1} u\right|^{*}(s) d s, \quad t>0 .
$$

For later purposes, we would like to observe that, using (8) , the reiteration theorem [6, Theorem V.2.4] and Theorem [2.2, if either $p_{0}=q_{0}=1$, or $1<p_{0}<p_{1}<\infty$ and $1 \leq q_{0}, q_{1}<\infty$, then, for any $t>0$,

$$
\text { (9) } \begin{aligned}
K\left(u, t ; W^{1} L^{p_{0}, q_{0}}\left(I^{n}\right), W^{1} L^{p_{1}, q_{1}}\left(I^{n}\right)\right) \approx & \left(\int_{0}^{t^{\alpha}}\left[s^{1 / p_{0}-1 / q_{0}}\left|D^{1} u\right|^{*}(s)\right]^{q_{0}} d s\right)^{1 / q_{0}} \\
& +t\left(\int_{t^{\alpha}}^{1}\left[s^{1 / p_{1}-1 / q_{1}}\left|D^{1} u\right|^{*}(s)\right]^{q_{1}} d s\right)^{1 / q_{1}},
\end{aligned}
$$

where $\alpha$ is defined as in Theorem 2.2. For several properties concerning Sobolev spaces, we refer to [1, 26, 11].

\section{MIXED NORM SPACES}

Our goal in this section is to present some basic properties of mixed norm spaces we shall need for our work (in what follows and throughout the paper we shall assume $n \geq 2$.)

Let $k \in\{1, \ldots, n\}$. We write $\widehat{x}_{k}$ for the point in $I^{n-1}$ obtained from a given vector $x \in I^{n}$ by removing its $k$ th coordinate. That is,

$$
\widehat{x}_{k}=\left(x_{1}, \ldots, x_{k-1}, x_{k+1}, \ldots, x_{n}\right) \in I^{n-1} .
$$

Moreover, for any $f \in \mathcal{M}\left(I^{n}\right)$, we use the notation $f_{\widehat{x}_{k}}$ for the function obtained from $f$, with $\widehat{x}_{k}$ fixed. Observe that, since $f$ is measurable, $f_{\widehat{x}_{k}}$ is also measurable a.e. $\widehat{x}_{k} \in I^{n-1}$.

We now recall the Benedek-Panzone spaces, which were introduced in [4] for the case of $L^{p}$. For further information on this topic see [13, 9, 10, 3].

Definition 3.1. Let $k \in\{1, \ldots, n\}$. Given two r.i. spaces $X\left(I^{n-1}\right)$ and $Y(I)$, the Benedek-Panzone space $\mathcal{R}_{k}(X, Y)$ is defined as the collection of all $f \in \mathcal{M}\left(I^{n}\right)$ satisfying

$$
\|f\|_{\mathcal{R}_{k}(X, Y)}=\left\|\psi_{k}(f, Y)\right\|_{X\left(I^{n-1}\right)}<\infty
$$

where $\psi_{k}(f, Y)\left(\widehat{x}_{k}\right)=\left\|f\left(\widehat{x}_{k}, \cdot\right)\right\|_{Y(I)}$.

Buhvalov [13] and Blozinski [9] proved that $\mathcal{R}_{k}(X, Y)$ is a Banach function space. Moreover, Boccuto, Bukhvalov, and Sambucini [10] proved that $\mathcal{R}_{k}(X, Y)$ is an r.i. space, if and only if $X=Y=L^{p}$, for some $1 \leq p \leq \infty$.

Now, we shall give the definition of the mixed norm spaces, sometimes also called symmetric mixed norm spaces. 
Definition 3.2. Given two r.i. spaces $X\left(I^{n-1}\right)$ and $Y(I)$, the mixed norm space $\mathcal{R}(X, Y)$ is defined as

$$
\mathcal{R}(X, Y)=\bigcap_{k=1}^{n} \mathcal{R}_{k}(X, Y) .
$$

For each $f \in \mathcal{R}(X, Y)$, we set $\|f\|_{\mathcal{R}(X, Y)}=\sum_{k=1}^{n}\|f\|_{\mathcal{R}_{k}(X, Y)}$.

It is not difficult to verify that $\mathcal{R}(X, Y)$ is a Banach function space. Since the pioneering works of Gagliardo [20, Nirenberg [28], and Fournier [19], many useful properties and generalizations of these spaces have been studied, via different methods, by various authors, including Blei [8], Milman [27], Algervik and Kolyada [2], and Kolyada [24, 25].

A thorough study of mixed norm spaces has been considered in [16]. In particular, extending the mixed norm estimates due to Fournier [19] to more general r.i. spaces, we were able to obtain a description of the smallest r.i. space that is continuously embedded into a fixed mixed norm space of the form $\mathcal{R}\left(X, L^{\infty}\right)$ (see [16, Theorem 5.6]):

Theorem 3.3. Let $X\left(I^{n-1}\right)$ be an r.i. space. Then, the space $Z_{\mathcal{R}\left(X, L^{\infty}\right)}\left(I^{n}\right)$ of all $f \in \mathcal{M}\left(I^{n}\right)$ such that

$$
\|f\|_{Z_{\mathcal{R}\left(X, L^{\infty}\right)}\left(I^{n}\right)}=\left\|f^{*}\left(t^{n^{\prime}}\right)\right\|_{\bar{X}(0,1)}<\infty,
$$

is an r.i. space. Moreover, $Z_{\mathcal{R}\left(X, L^{\infty}\right)}\left(I^{n}\right)$ is the smallest r.i. space satisfying

$$
\mathcal{R}\left(X, L^{\infty}\right) \hookrightarrow Z_{\mathcal{R}\left(X, L^{\infty}\right)}\left(I^{n}\right)
$$

holds.

Furthermore, we can see that (10) is a strict embedding when

$$
Z\left(I^{n}\right) \neq L^{\infty}\left(I^{n}\right)=\mathcal{R}\left(L^{\infty}, L^{\infty}\right) .
$$

Theorem 3.4. Let $X\left(I^{n-1}\right)$ and $Z\left(I^{n}\right)$ be r.i. spaces. Then,

$$
Z\left(I^{n}\right) \hookrightarrow \mathcal{R}\left(X, L^{\infty}\right) \Longleftrightarrow Z\left(I^{n}\right)=L^{\infty}\left(I^{n}\right) .
$$

To prove this, we first need to recall a result concerning embeddings between mixed norms (see [16, Theorem 4.6]).

Theorem 3.5. Let $X_{1}\left(I^{n-1}\right)$ and $X_{2}\left(I^{n-1}\right)$ be r.i. spaces. Then,

$$
\mathcal{R}\left(X_{1}, L^{\infty}\right) \hookrightarrow \mathcal{R}\left(X_{2}, L^{\infty}\right) \Longleftrightarrow X_{1}\left(I^{n-1}\right) \hookrightarrow X_{2}\left(I^{n-1}\right) .
$$

Proof of Theorem 3.4. In view of Theorem 3.5, it suffices to prove the necessary part of this result. We shall see that if

$$
L^{\infty}\left(I^{n}\right) \not z Z\left(I^{n}\right)
$$

then

$$
Z\left(I^{n}\right) \hookrightarrow \mathcal{R}\left(X, L^{\infty}\right) .
$$

We may suppose, without loss of generality, that $I=(-a, b)$, with $a, b \in \mathbb{R}_{+}$. Let $0<r<\min (a, b)$. Given any function $g \in Z\left(I^{n}\right)$, but $g \notin L^{\infty}\left(I^{n}\right)$, we define

$$
f(x)= \begin{cases}g^{*}\left(2\left|x_{n}\right|\right), & \text { if }\left(\widehat{x}_{n}, x_{n}\right) \in I^{n-1} \times(-r, r), \\ 0, & \text { otherwise. }\end{cases}
$$


Let us see that $f \in Z\left(I^{n}\right)$ and $f \notin \mathcal{R}\left(X, L^{\infty}\right)$. In fact, using (6) , we get

$$
f^{*}(t)= \begin{cases}g^{*}(t), & \text { if } 0 \leq t<\min \left(2 r, \lambda_{g}(0)\right), \\ 0, & \text { otherwise. }\end{cases}
$$

Hence, our assumption on $g$ ensures that

$$
\|f\|_{Z\left(I^{n}\right)} \leq\|g\|_{Z\left(I^{n}\right)}<\infty .
$$

On the other hand, for any $\widehat{x}_{n} \in I^{n-1}$, it holds that

$$
\psi_{n}\left(f, L^{\infty}\right)\left(\widehat{x}_{n}\right)=\|g\|_{L^{\infty}(I)}=\infty .
$$

Hence $f \notin \mathcal{R}_{n}\left(X, L^{\infty}\right)$ and the proof is complete.

Taking into account Theorem 3.4, it is immediate to see that a mixed norm space $\mathcal{R}\left(X, L^{\infty}\right)$ is an r.i. space if and only if $\mathcal{R}\left(X, L^{\infty}\right)=L^{\infty}\left(I^{n}\right)$, which is equivalent to $X\left(I^{n-1}\right)=L^{\infty}\left(I^{n-1}\right)$.

We end this section by recalling the expression of the $K$-functional for the couple of mixed norm spaces $\left(\mathcal{R}\left(X, L^{\infty}\right), L^{\infty}\right)$ given in [16].

Theorem 3.6. Let $X\left(I^{n-1}\right)$ be an r.i. space and let $f \in \mathcal{R}\left(X, L^{\infty}\right)+L^{\infty}\left(I^{n}\right)$. Then,

$$
K\left(f, \varphi_{X}(t), \mathcal{R}\left(X, L^{\infty}\right), L^{\infty}\right) \approx \sum_{k=1}^{n}\left\|\psi_{k}^{*}\left(f, L^{\infty}\right) \chi_{(0, t)}\right\|_{\bar{X}(0,)}, \quad t>0,
$$

where $\varphi_{X}(t)$ is the fundamental function of $X\left(I^{n-1}\right)$ defined in (7).

\section{Sobolev EMBEDdings IN MIXED NORM SPACES}

Our aim in this section is to study the Sobolev embedding of the form

$$
W^{1} Z\left(I^{n}\right) \hookrightarrow \mathcal{R}\left(X, L^{\infty}\right),
$$

extending the classical estimate (2) proved by Gagliardo [20] and Nirenberg [28]. In particular, we are interested in the following problems:

(i) We would like to find the smallest space of the form $\mathcal{R}\left(X, L^{\infty}\right)$ in (11), for a given r.i. $Z\left(I^{n}\right)$.

(ii) On the other hand, given a fixed range space $\mathcal{R}\left(X, L^{\infty}\right)$, we would like to provide a characterization of the largest r.i. domain space satisfying (11).

4.1. Necessary and sufficient conditions. Now, our main purpose is to find necessary and sufficient conditions on $X\left(I^{n-1}\right)$ and $Z\left(I^{n}\right)$ under which we have the embedding (11).

For this, we shall establish the equivalence between (11) and the boundedness of a Hardy type operator, via an argument used by Kerman and Pick [23] to characterize Sobolev embeddings in r.i. spaces. Then, this relation will be a key tool in determining the largest r.i. space and the smallest mixed norm space for (11).

Let us start with an auxiliary lemma. The proof, based on a classical interpolation result due to Calderón (see [6, Theorem III.2.12]), follows the scheme of [15, Lemma 4.1], so we do not include it here.

Lemma 4.1. Let $\beta>-1$ and let $Y(0,1)$ be an r.i. space. Then,

$$
\left\|\int_{t}^{1} s^{\beta} f(s) d s\right\|_{Y(0,1)} \lesssim\|f\|_{Y(0,1)}, f \in Y(0,1) .
$$


Theorem 4.2. Let $X\left(I^{n-1}\right)$ and $Z\left(I^{n}\right)$ be r.i. spaces. Then, the following statements are equivalent:

(i) $W^{1} Z\left(I^{n}\right) \hookrightarrow \mathcal{R}\left(X, L^{\infty}\right)$;

(ii) $\left\|\int_{t^{n^{\prime}}}^{1} f^{*}(s) s^{-1 / n^{\prime}} d s\right\|_{\bar{X}(0,1)} \lesssim\left\|f^{*}\right\|_{\bar{Z}(0,1)}, \quad f \in Z\left(I^{n}\right)$.

Proof. First we prove that $(i) \Rightarrow(i i)$. As in the proof of Theorem 3.4, we may assume that $I=(-a, b)$, with $a, b \in \mathbb{R}_{+}$and $0<r<\min (a, b)$. Given any function $f \in Z\left(I^{n}\right)$, with $\lambda_{f}(0) \leq \omega_{n-1}^{n^{\prime}} r^{n}$, we define

$$
u(x)= \begin{cases}\int_{\omega_{n-1}^{n^{\prime}}|x|^{n}}^{\omega_{n-1}^{n^{\prime}} r^{n}} s^{-1 / n^{\prime}} f^{*}(s) d s, & \text { if } x \in B_{n}(0, r), \\ 0, & \text { otherwise. }\end{cases}
$$

Then, by (6) and the boundedness of the dilation operator in r.i. spaces, we get

$$
\|u\|_{Z\left(I^{n}\right)} \lesssim\left\|\int_{t}^{I^{n}} s^{-1 / n^{\prime}} f^{*}(s) d s\right\|_{\bar{Z}(0,1)},
$$

and so, Lemma 4.1 gives

$$
\|u\|_{Z\left(I^{n}\right)} \lesssim\left\|f^{*}\right\|_{\bar{Z}(0,1)}
$$

On the other hand, we have

$$
|\nabla u(x)| \approx f^{*}\left(\omega_{n-1}^{n^{\prime}}|x|^{n}\right), \text { a.e. } x \in B_{n}(0, r),
$$

and $|\nabla u(x)|=0$ otherwise. So, using again the boundedness of the dilation operator in r.i. spaces, we get

$$
\||\nabla u|\|_{Z\left(I^{n}\right)} \lesssim\left\|f^{*}\right\|_{\bar{Z}(0,1)} .
$$

By hypothesis $f \in Z\left(I^{n}\right)$, so inequalities (12) and (13) imply that $u \in W^{1} Z\left(I^{n}\right)$ and

$$
\|u\|_{W^{1} Z\left(I^{n}\right)}=\|u\|_{Z\left(I^{n}\right)}+\|\mid \nabla u\|_{Z\left(I^{n}\right)} \lesssim\left\|f^{*}\right\|_{\bar{Z}(0,1)} .
$$

Therefore, using $W^{1} Z\left(I^{n}\right) \hookrightarrow \mathcal{R}\left(X, L^{\infty}\right)$, we obtain

$$
\|u\|_{\mathcal{R}\left(X, L^{\infty}\right)} \lesssim\left\|f^{*}\right\|_{\bar{Z}(0,1)} .
$$

Now, let us compute $\|u\|_{\mathcal{R}\left(X, L^{\infty}\right)}$. For this, we fix any $k \in\{1, \ldots, n\}$. Then, we have

$$
\psi_{k}\left(u, L^{\infty}\right)\left(\widehat{x}_{k}\right)= \begin{cases}\int_{\omega_{n-1}^{n^{\prime}}\left|\widehat{x}_{k}\right|^{n}}^{\omega_{n-1}^{n^{\prime}} r^{n}} s^{-1 / n^{\prime}} f^{*}(s) d s, & \text { if } \widehat{x}_{k} \in B_{n-1}(0, r), \\ 0, & \text { otherwise. }\end{cases}
$$

As a consequence, using again ([6), we get

$$
\|u\|_{\mathcal{R}\left(X, L^{\infty}\right)}=\sum_{k=1}^{n}\left\|\psi_{k}^{*}\left(u, L^{\infty}\right)\right\|_{\bar{X}(0,1)}=n\left\|\int_{t^{n^{\prime}}}^{1} s^{-1 / n^{\prime}} f^{*}(s) d s\right\|_{\bar{X}(0,1)} .
$$

Thus, using (14), we obtain

$$
\left\|\int_{t^{n^{\prime}}}^{1} s^{-1 / n^{\prime}} f^{*}(s) d s\right\|_{\bar{X}(0,1)} \lesssim\|f\|_{\bar{Z}(0,1)} .
$$


This proves $(i i)$, for any $f \in Z\left(I^{n}\right)$, with $\lambda_{f}(0) \leq \omega_{n-1}^{n^{\prime}} r^{n}$. Now, let us consider any $f \in Z\left(I^{n}\right)$. We define

$$
f_{1}(x)=\max \left(|f(x)|-f^{*}\left(\omega_{n-1}^{n^{\prime}} r^{n}\right), 0\right) \operatorname{sgn} f(x),
$$

and

$$
f_{2}(x)=\min \left(|f(x)|, f^{*}\left(\omega_{n-1}^{n^{\prime}} r^{n}\right)\right) \operatorname{sgn} f(x) .
$$

We observe that $f=f_{1}+f_{2}$,

$$
f_{1}^{*}(t)= \begin{cases}f^{*}(t)-f^{*}\left(\omega_{n-1}^{n^{\prime}} r^{n}\right), & 0 \leq t<\lambda_{f}\left(f^{*}\left(\omega_{n-1}^{n^{\prime}} r^{n}\right)\right) \\ 0, & \text { otherwise }\end{cases}
$$

and

$$
f_{2}^{*}(t)= \begin{cases}f^{*}\left(\omega_{n-1}^{n^{\prime}} r^{n}\right), & 0 \leq t<\lambda_{f}\left(f^{*}\left(\omega_{n-1}^{n^{\prime}} r^{n}\right)\right), \\ f^{*}(t), & \text { otherwise. }\end{cases}
$$

So, combining (16) and (17), we have $f^{*}=f_{1}^{*}+f_{2}^{*}$. Using now inequality (15), with $f$ replaced by $f_{1}$, we get

$$
\left\|\int_{t^{n^{\prime}}}^{1} s^{-1 / n^{\prime}} f_{1}^{*}(s) d s\right\|_{\bar{X}(0,1)} \lesssim\left\|f^{*}\right\|_{Z(0,1)} .
$$

On the other hand, by Hölder's inequality, we have

$$
\left\|\int_{t^{n^{\prime}}}^{1} s^{-1 / n^{\prime}} f_{2}^{*}(s) d s\right\|_{\bar{X}(0,1)} \lesssim f^{* *}\left(\omega_{n-1}^{n^{\prime}} r^{n}\right) \lesssim\|f\|_{Z(0,1)} .
$$

As a consequence, using (18) and (19), we get

$$
\begin{aligned}
\left\|\int_{t^{n^{\prime}}}^{1} s^{-1 / n^{\prime}} f^{*}(s) d s\right\|_{\bar{X}(0,1)} & =\left\|\int_{t^{n^{\prime}}}^{1} s^{-1 / n^{\prime}}\left(f_{1}^{*}(s)+f_{2}^{*}(s)\right) d s\right\|_{\bar{X}(0,1)} \\
\leq & \left\|\int_{t^{n^{\prime}}}^{1} s^{-1 / n^{\prime}} f_{1}^{*}(s) d s\right\|_{\bar{X}(0,1)} \\
& +\left\|\int_{t^{n^{\prime}}}^{1} s^{-1 / n^{\prime}} f_{2}^{*}(s) d s\right\|_{\bar{X}(0,1)} \lesssim\left\|f^{*}\right\|_{Z(0,1)},
\end{aligned}
$$

which is $(i i)$.

Conversely, let us suppose that (ii) holds. We fix any $f \in W^{1} Z\left(I^{n}\right)$. Combining the classical embedding on Lorentz spaces (cf. e.g. [29, 33])

$$
W^{1} L^{n, 1}\left(I^{n}\right) \hookrightarrow \mathcal{R}\left(L^{\infty}, L^{\infty}\right)=L^{\infty}\left(I^{n}\right),
$$

with Gagliardo-Nirenberg embedding (2), and then applying Theorem 2.1, we get

$$
K\left(f, t ; \mathcal{R}\left(L^{1}, L^{\infty}\right), L^{\infty}\right) \lesssim K\left(f, C t ; W^{1} L^{1}, W^{1} L^{n, 1}\right), \quad 0<t<1 .
$$

We have, by Theorem 3.6,

$$
K\left(f, t ; \mathcal{R}\left(L^{1}, L^{\infty}\right), L^{\infty}\right) \approx \sum_{k=1}^{n} \int_{0}^{t} \psi_{k}^{*}\left(f, L^{\infty}\right)(s) d s .
$$


Moreover, using now (9), we get

$$
\begin{aligned}
K\left(f, C t ; W^{1} L^{1}, W^{1} L^{n, 1}\right) & \approx \int_{0}^{(C t)^{n^{\prime}}}\left|D^{1} f\right|^{*}(s) d s+C t \int_{(C t)^{n^{\prime}}}^{1} s^{-1 / n^{\prime}}\left|D^{1} f\right|^{*}(s) d s \\
& \approx \int_{0}^{(C t)^{n^{\prime}}} s^{-1 / n}\left(\int_{s}^{1} y^{-1 / n^{\prime}}\left|D^{1} f\right|^{*}(y) d y\right) d s .
\end{aligned}
$$

So, by a change of variables, we obtain

$$
K\left(f, C t ; W^{1} L^{1}, W^{1} L^{n, 1}\right) \approx \int_{0}^{t}\left(\int_{C s^{n^{\prime}}}^{1} y^{-1 / n^{\prime}}\left|D^{1} f\right|^{*}(y) d y\right) d s .
$$

Therefore, taking into account (20), (21), and (22), we obtain

$$
\int_{0}^{t} \psi_{k}^{*}\left(f, L^{\infty}\right)(s) d s \lesssim \int_{0}^{t}\left(\int_{C s^{n^{\prime}}}^{1} y^{-1 / n^{\prime}}\left|D^{1} f\right|^{*}(y) d y\right) d s, \quad k \in\{1, \ldots, n\} .
$$

So, using Hardy-Littlewood-Pólya Principle, the boundedness of the dilation operator in r.i. spaces and (ii), we get

$$
\|f\|_{\mathcal{R}_{k}\left(X, L^{\infty}\right)} \lesssim\left\|\int_{s^{n^{\prime}}}^{1} y^{-1 / n^{\prime}}\left|D^{1} f\right|^{*}(y) d y\right\|_{\bar{X}(0,1)} \lesssim\left\|\left|D^{1} f\right|^{*}\right\|_{\bar{Z}(0,1)} \approx\|f\|_{W^{1} Z\left(I^{n}\right)},
$$

for any $k \in\{1, \ldots, n\}$, from which $(i)$ follows.

Remark 4.3. Using a duality argument, we observe that the statements proved in Theorem 4.2 are also equivalent to the following additional condition:

$$
\sup _{\|f\|_{X^{\prime}\left(I^{n-1}\right)} \leq 1}\left\|f^{* *}\left(t^{1 / n^{\prime}}\right)\right\|_{\bar{Z}^{\prime}(0,1)}=\sup _{\|g\|_{Z_{\left(I^{n}\right)} \leq 1}}\left\|\int_{s^{n^{\prime}}}^{1} g^{*}(t) t^{-1 / n^{\prime}} d t\right\|_{\bar{X}(0,1)}<\infty .
$$

In fact, we fix any $f \in X^{\prime}\left(I^{n-1}\right)$, with $\|f\|_{X^{\prime}\left(I^{n-1}\right)} \leq 1$. Then, by Fubini's theorem and Hölder's inequality, we get

$$
\begin{aligned}
\left\|f^{* *}\left(t^{1 / n^{\prime}}\right)\right\|_{\bar{Z}^{\prime}(0,1)} & =\sup _{\|g\|_{Z\left(I^{n}\right) \leq 1}} \int_{0}^{1} f^{*}(t)\left(\int_{t^{n^{\prime}}}^{1} s^{-1 / n^{\prime}} g^{*}(s) d s\right) d t \\
& \leq \sup _{\|g\|_{Z\left(I^{n}\right) \leq 1}}\|f\|_{X^{\prime}\left(I^{n-1}\right)}\left\|\int_{t^{n^{\prime}}}^{1} s^{-1 / n^{\prime}} g^{*}(s) d s\right\|_{\bar{X}(0,1)} \\
& \leq \sup _{\|g\|_{Z\left(I^{n}\right) \leq 1}}\left\|\int_{t^{n^{\prime}}}^{1} s^{-1 / n^{\prime}} g^{*}(s) d s\right\|_{\bar{X}(0,1)} .
\end{aligned}
$$

Therefore, we conclude that

$$
\sup _{\|f\|_{X^{\prime}\left(I^{n-1}\right)} \leq 1}\left\|f^{* *}\left(t^{1 / n^{\prime}}\right)\right\|_{\bar{Z}^{\prime}(0,1)} \leq \sup _{\|g\|_{Z\left(I^{n}\right)} \leq 1}\left\|\int_{t^{n^{\prime}}}^{1} s^{-1 / n^{\prime}} g^{*}(s) d s\right\|_{\bar{X}(0,1)} .
$$

Applying the same arguments as before, we obtain the converse inequality. 
4.2. Characterization of the optimal range. Now, we fix an r.i. space $Z\left(I^{n}\right)$. We shall provide a description of the smallest space of the form $\mathcal{R}\left(X, L^{\infty}\right)$ satisfying

$$
W^{1} Z\left(I^{n}\right) \hookrightarrow \mathcal{R}\left(X, L^{\infty}\right) .
$$

It is important to note that Theorem 4.2, together with Remark 4.3, relate this problem with that of finding the largest r.i. space $Y\left(I^{n-1}\right)$ such that

$$
H^{\prime}: Y\left(I^{n-1}\right) \rightarrow \bar{Z}^{\prime}(0,1)
$$

is bounded, where $H^{\prime}$ is the conjugate Hardy type operator:

$$
H^{\prime} f(t)=f^{* *}\left(t^{1 / n^{\prime}}\right), \quad f \in \mathcal{M}\left(I^{n-1}\right) .
$$

Hence, it is natural to introduce a new space, denoted by $Y\left(I^{n-1}\right)$, consisting of all $f \in \mathcal{M}\left(I^{n-1}\right)$ such that

$$
\|f\|_{Y\left(I^{n-1}\right)}=\left\|f^{* *}\left(t^{-1 / n^{\prime}}\right)\right\|_{\bar{Z}^{\prime}(0,1)}<\infty .
$$

It is not difficult to verify that $Y\left(I^{n-1}\right)$ is an r.i. space equipped with the norm $\|\cdot\|_{Y\left(I^{n-1}\right)}$. Hence, using (23) , (24) , and a duality argument, we have that its associate space $Y^{\prime}\left(I^{n-1}\right)$ verifies that

$$
H: \bar{Z}(0,1) \rightarrow \bar{Y}^{\prime}\left(I^{n-1}\right),
$$

where $H$ is the Hardy type operator:

$$
H f(t)=\int_{t^{n^{\prime}}}^{1} s^{-1 / n^{\prime}} f^{*}(s) d s, \quad f \in \mathcal{M}\left(I^{n}\right) .
$$

In order to clarify the notation used later, note that if we denote by

$$
X_{W^{1} Z, L^{\infty}}\left(I^{n-1}\right):=Y^{\prime}\left(I^{n-1}\right),
$$

then, [6, Theorem I.2.7] implies that

$$
Y\left(I^{n-1}\right)=\left(Y^{\prime}\right)^{\prime}\left(I^{n-1}\right)=X_{W^{1} Z, L^{\infty}}^{\prime}\left(I^{n-1}\right) .
$$

Theorem 4.4. Let $Z\left(I^{n}\right)$ be an r.i. space and let $X_{W^{1} Z, L^{\infty}}\left(I^{n-1}\right)$ be the r.i. space defined in (27). Then, the Sobolev embedding

$$
W^{1} Z\left(I^{n}\right) \hookrightarrow \mathcal{R}\left(X_{W^{1} Z, L^{\infty}}, L^{\infty}\right),
$$

holds. Moreover, $\mathcal{R}\left(X_{W^{1} Z, L^{\infty}}, L^{\infty}\right)$ is the smallest space of the form $\mathcal{R}\left(X, L^{\infty}\right)$ that verifies (28).

Proof. The embedding (28) follows directly from Theorem 4.2 together with (25)). Thus, to complete the proof, it only remains to see that $\mathcal{R}\left(X_{W^{1} Z, L^{\infty}}, L^{\infty}\right)$ is the smallest space of the form $\mathcal{R}\left(X, L^{\infty}\right)$ satisfying (28). Hence, we shall see that if a mixed norm space $\mathcal{R}\left(X, L^{\infty}\right)$ verifies

$$
W^{1} Z\left(I^{n}\right) \hookrightarrow \mathcal{R}\left(X, L^{\infty}\right),
$$

then

$$
\mathcal{R}\left(X_{W^{1} Z, L^{\infty}}, L^{\infty}\right) \hookrightarrow \mathcal{R}\left(X, L^{\infty}\right) .
$$

We fix any $g \in X^{\prime}\left(I^{n-1}\right)$. Then, combining (29) with Remark 4.3, we get

$$
\left\|g^{* *}\left(t^{1 / n^{\prime}}\right)\right\|_{\bar{Z}^{\prime}(0,1)} \lesssim\|g\|_{X^{\prime}\left(I^{n-1}\right)}
$$


Therefore, using now (24), we obtain

$$
X^{\prime}\left(I^{n-1}\right) \hookrightarrow X_{W^{1} Z, L^{\infty}}^{\prime}\left(I^{n-1}\right) .
$$

As a consequence, [6, Proposition I.2.10] and Theorem 3.5 imply that (28) holds, as we wanted to show.

Now, we shall present some applications of Theorem 4.4. In particular, we shall see that (2) cannot be improved within the class of spaces of the form $\mathcal{R}\left(X, L^{\infty}\right)$. This should be understood as follows: if we replace the range space in

$$
W^{1} L^{1}\left(I^{n}\right) \hookrightarrow \mathcal{R}\left(L^{1}, L^{\infty}\right),
$$

by a smaller mixed norm space, say $\mathcal{R}\left(X, L^{\infty}\right)$, then the resulting embedding

$$
W^{1} L^{1}\left(I^{n}\right) \hookrightarrow \mathcal{R}\left(X, L^{\infty}\right)
$$

cannot longer be true.

Corollary 4.5. Let $1 \leq p<n$. Then, the mixed norm space $\mathcal{R}\left(L^{p(n-1) /(n-p), p}, L^{\infty}\right)$ is the smallest space of the form $\mathcal{R}\left(X, L^{\infty}\right)$ satisfying

$$
W^{1} L^{p}\left(I^{n}\right) \hookrightarrow \mathcal{R}\left(L^{p(n-1) /(n-p), p}, L^{\infty}\right) .
$$

Proof. We prove this result only when $1<p<n$ ( $p=1$ is easier). Theorem 4.4, with $Z\left(I^{n}\right)$ replaced by $L^{p}\left(I^{n}\right)$, gives

$$
\begin{aligned}
\|f\|_{X_{W^{1} L^{p}, L^{\infty}}^{\prime}\left(I^{n-1}\right)} & =\left\|f^{* *}\left(t^{1 / n^{\prime}}\right)\right\|_{L^{p^{\prime}}(0,1)} \\
& \approx\left(\int_{0}^{1} t^{-(n-p) /((n-1)(p-1))-1}\left(\int_{0}^{t} f^{*}(s) d s\right)^{p^{\prime}} d t\right)^{1 / p^{\prime}} .
\end{aligned}
$$

Since $1<p<n$, we may apply Hardy's inequalities [6, Lemma III.3.9] to get

$$
\|f\|_{X_{W^{1} L^{p}, L^{\infty}}^{\prime}\left(I^{n-1}\right)} \lesssim\|f\|_{L^{p^{\prime}(n-1) / n, p^{\prime}\left(I^{n-1}\right)}} .
$$

On the other hand, we have

$$
\|f\|_{X_{W^{1} L^{p}, L^{\infty}}^{\prime}\left(I^{n-1}\right)} \gtrsim\|f\|_{L^{p^{\prime}(n-1) / n, p^{\prime}\left(I^{n-1}\right)}} .
$$

As a consequence, combining (30) and (31), we get

$$
X_{W^{1} L^{p}, L^{\infty}}^{\prime}\left(I^{n-1}\right)=L^{p^{\prime}(n-1) / n, p^{\prime}}\left(I^{n-1}\right),
$$

and hence, using [6, Theorem IV.4.7], we have that

$$
X_{W^{1} L^{p}, L^{\infty}}\left(I^{n-1}\right)=L^{p(n-1) /(n-p), p}\left(I^{n-1}\right),
$$

from which the result follows.

Now, we shall apply Theorem 4.4 to the so-called limiting or critical case of the classical Sobolev embedding.

Corollary 4.6. The mixed norm space $\mathcal{R}\left(L^{\infty, n ;-1}, L^{\infty}\right)$ is the smallest space of the form $\mathcal{R}\left(X, L^{\infty}\right)$ satisfying

$$
W^{1} L^{n}\left(I^{n}\right) \hookrightarrow \mathcal{R}\left(L^{\infty, n ;-1}, L^{\infty}\right) .
$$

The proof will be an immediate consequence of Theorem 4.4 and the following result given in [18].

Theorem 4.7. Let $1<p<\infty$ and let $v$ be a weight on $(0,1)$ satisfying the following properties: 
(i) $\int_{0}^{1} v(t) d t<\infty$;

(ii) $\int_{0}^{1} t^{-p} v(t)^{p} d t=\infty$;

(iii) $\int_{0}^{r} v(t)^{p} d t \lesssim r^{p}\left(1+\int_{r}^{1} t^{-p} v(t)^{p} d t\right), 0<r<1$.

Then, the r.i. norm defined as

$$
\|f\|_{X(0,1)}=\left\|v(t) f^{* *}(t)\right\|_{L^{p}(0,1)}, \quad f \in \mathcal{M}(0,1)
$$

has associate norm

$$
\|g\|_{X^{\prime}(0,1)}=\left\|w(t) g^{*}(t)\right\|_{L^{p^{\prime}(0,1)}}, \quad g \in \mathcal{M}(0,1),
$$

where

$$
w(t)^{p^{\prime}}=\frac{d}{d t}\left[\left(1+\int_{t}^{1} s^{-p} v(s)^{p} d s\right)^{1-p^{\prime}}\right], \quad 0<t<1 .
$$

Proof of Corollary 4.6. By Theorem 4.4, with $Z\left(I^{n}\right)=L^{n}\left(I^{n}\right)$, we get

$$
\|f\|_{X_{W^{1} L^{n}, L^{\infty}}^{\prime}\left(I^{n-1}\right)}=\left\|f^{* *}\left(t^{1 / n^{\prime}}\right)\right\|_{L^{n^{\prime}\left(I^{n}\right)}} .
$$

Consequently, Theorem 4.7 implies that

$$
\|f\|_{X_{W^{1} L^{n}, L^{\infty}}\left(I^{n-1}\right)}=\left\|t^{-1} \log (e / t) f^{*}(s) d s\right\|_{L^{n}\left(I^{n-1}\right)},
$$

from which it follows that

$$
\mathcal{R}\left(X_{W^{1} L^{n}, L^{\infty}} L^{\infty}\right)=\mathcal{R}\left(L^{\infty, n ;-1}, L^{\infty}\right),
$$

as we wanted to prove.

4.3. Characterization of the optimal domain. We now focus on the problem of determining the largest r.i. domain space satisfying (11) for a fixed range space $\mathcal{R}\left(X, L^{\infty}\right)$. Observe that the equivalences proved in Theorem 4.2 suggest that in order to solve this problem, we should find the largest r.i. space $Z\left(I^{n}\right)$ such that

$$
H: Z\left(I^{n}\right) \rightarrow \bar{X}(0,1)
$$

is bounded, where $H$ is the Hardy type operator defined in (26). Hence, it is natural to consider a new space, denoted by $Z_{\mathcal{R}\left(X, L^{\infty}\right)}\left(I^{n}\right)$, consisting of all $f \in \mathcal{M}\left(I^{n}\right)$ for which

$$
\|f\|_{\left.Z_{\mathcal{R}(X, L}\right)}=\left\|\int_{t^{n^{\prime}}}^{1} f^{* *}(s) s^{-1 / n^{\prime}} d s\right\|_{\bar{X}(0,1)}<\infty .
$$

It is not difficult to verify that $Z_{\mathcal{R}\left(X, L^{\infty}\right)}\left(I^{n}\right)$ is an r.i. space equipped with the norm $\|\cdot\|_{Z_{\mathcal{R}\left(X, L^{\infty}\right)}\left(I^{n}\right)}$. The next lemma will be needed later. Its proof follows the same arguments used in [18, Theorem 4.4], with small modifications, and hence we will omit it.

Lemma 4.8. Let $X\left(I^{n-1}\right)$ be an r.i space, with $\bar{\alpha}_{X}<1$. Then,

$$
\|f\|_{Z_{\mathcal{R}\left(X, L^{\infty}\right)}\left(I^{n}\right)} \approx\left\|\int_{t^{n^{\prime}}}^{1} f^{*}(s) s^{-1 / n^{\prime}} d s\right\|_{\bar{X}(0,1)}, \quad f \in \mathcal{M}\left(I^{n}\right) .
$$


Theorem 4.9. Let $X\left(I^{n-1}\right)$ be an r.i space, $\bar{\alpha}_{X}<1$, and let $Z_{\mathcal{R}\left(X, L^{\infty}\right)}\left(I^{n}\right)$ be the r.i. space defined in (33). Then, the Sobolev embedding

$$
W^{1} Z_{\mathcal{R}\left(X, L^{\infty}\right)}\left(I^{n}\right) \hookrightarrow \mathcal{R}\left(X, L^{\infty}\right)
$$

holds. Moreover, $Z_{\mathcal{R}\left(X, L^{\infty}\right)}\left(I^{n}\right)$ is the largest domain space for which (34) holds.

Proof. Theorem 4.2 ensures us that (34) holds. Hence, to complete the proof, it only remains to show that $Z_{\mathcal{R}\left(X, L^{\infty}\right)}\left(I^{n}\right)$ is the largest r.i. space satisfying (34). We shall see that if another space, namely $Z\left(I^{n}\right)$, verifies

$$
W^{1} Z\left(I^{n}\right) \hookrightarrow \mathcal{R}\left(X, L^{\infty}\right)
$$

then

$$
Z\left(I^{n}\right) \hookrightarrow Z_{\mathcal{R}\left(X, L^{\infty}\right)}\left(I^{n}\right)
$$

We fix any $f \in Z\left(I^{n}\right)$. Then, using again Theorem 4.2, we get

$$
\left\|\int_{t^{n^{\prime}}}^{1} f^{*}(s) s^{-1 / n^{\prime}} d s\right\|_{\bar{X}(0,1)} \lesssim\left\|f^{*}\right\|_{\bar{Z}(0,1)} .
$$

But, by Lemma 4.8, we have

$$
\|f\|_{Z_{\mathcal{R}\left(X, L^{\infty}\right)\left(I^{n}\right)}} \approx\left\|\int_{t^{n^{\prime}}}^{1} s^{-1 / n^{\prime}} f^{*}(s) d s\right\|_{\bar{X}(0,1)},
$$

and hence, using (35), the result follows.

Now, we shall present some applications of Theorem 4.9.

Corollary 4.10. Let $1<p<n$. Then, the Lebesgue space $L^{p}\left(I^{n}\right)$ is the largest r.i. space satisfying

$$
W^{1} L^{p}\left(I^{n}\right) \hookrightarrow \mathcal{R}\left(L^{p(n-1) /(n-p), p}, L^{\infty}\right) .
$$

Proof. If $X\left(I^{n-1}\right)=L^{p(n-1) /(n-p), p}\left(I^{n-1}\right)$ then, by Theorem 4.9, we obtain that

$$
\begin{aligned}
\|f\|_{Z_{\mathcal{R}\left(L^{p(n-1) /(n-p), p}, L^{\infty}\right)}\left(I^{n}\right)} & \approx\left\|\int_{t^{n^{\prime}}}^{1} f^{*}(s) s^{-1 / n^{\prime}} d s\right\|_{L^{p(n-1) /(n-p), p}(0,1)} \\
& \approx\left(\int_{0}^{1} t^{(n-p) / n-1}\left(\int_{t}^{1} f^{*}(s) s^{-1 / n^{\prime}} d s\right)^{p} d t\right)^{1 / p} .
\end{aligned}
$$

Using now Hardy's inequalities we obtain

$$
\|f\|_{Z_{\mathcal{R}\left(L^{p(n-1) /(n-p), p, L \infty)}\right.}\left(I^{n}\right)} \lesssim\|f\|_{L^{p}\left(I^{n}\right)}
$$

On the other hand, again Hardy's inequalities give us that

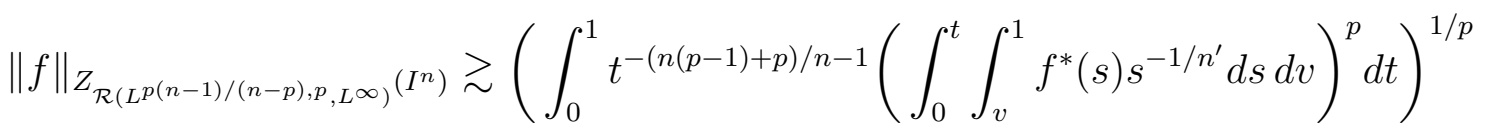

$$
\begin{aligned}
& =\left(\int_{0}^{1} t^{-p(n-1) / n}\left(\int_{0}^{t} \int_{v}^{1} f^{*}(s) s^{-1 / n^{\prime}} d s d v\right)^{p} d t\right)^{1 / p} .
\end{aligned}
$$

But, if $0<t<1$, then

$$
\int_{0}^{t} \int_{v}^{1} f^{*}(s) s^{-1 / n^{\prime}} d s d v=t \int_{t}^{1} f^{*}(s) s^{-1 / n^{\prime}} d s+\int_{0}^{t} f^{*}(v) v^{1 / n} d v \gtrsim f^{*}(t) t^{1 / n+1} .
$$


So,

$$
\|f\|_{Z_{\mathcal{R}\left(L^{\left.p(n-1) /(n-m p), p, L^{\infty}\right)}\right.}\left(I^{n}\right)} \gtrsim\|f\|_{L^{p}\left(I^{n}\right)} .
$$

Thus, combining (36) and (37), we obtain

$$
L^{p}\left(I^{n}\right)=Z_{\mathcal{R}\left(L^{p(n-1) /(n-p), p}, L^{\infty}\right)}\left(I^{n}\right),
$$

as we wanted to show.

Finally, we shall see that a nontrivial improvement of the domain in (32) is possible among r.i. spaces. For this, we will use the following result [5]:

Lemma 4.11. If $1<p<\infty$, then $\bar{\alpha}_{L^{\infty, p ;-1}}=0$.

Corollary 4.12. The r.i. space $Z_{\mathcal{R}\left(L^{\infty, n ;-1}, L^{\infty}\right)}\left(I^{n}\right)$, with norm given by

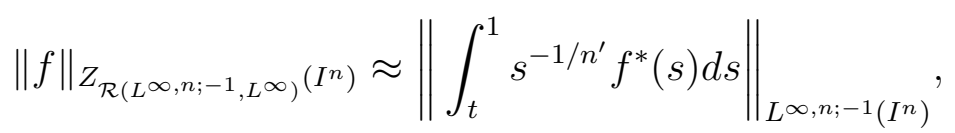

is the largest r.i. domain space that verifies

$$
W^{1} Z_{\mathcal{R}\left(L^{\infty, n ;-1}, L^{\infty}\right)}\left(I^{n}\right) \hookrightarrow \mathcal{R}\left(L^{\infty, n ;-1}, L^{\infty}\right) .
$$

Proof. According to Lemma 4.11, we may apply Theorem 4.9 to obtain

$$
\|f\|_{Z_{\mathcal{R}\left(L^{\infty, n ;-1, L}\right)}\left(I^{n}\right)} \approx\left\|\int_{t^{n^{\prime}}}^{1} s^{-1 / n^{\prime}} f^{*}(s) d s\right\|_{L^{\infty, n ;-1}\left(I^{n}\right)} .
$$

Then, the result follows using a change of variables and [14, Theorem 3.1].

\section{Comparison With the optimal R.i. Space}

As we have mentioned before, Kerman and Pick [23] studied the optimal range problem for Sobolev embedding within the class of r.i. spaces. Namely, for a fixed r.i. domain space $Z\left(I^{n}\right)$, they determined the smallest r.i. space $X^{\mathrm{op}}\left(I^{n}\right)$, satisfying

$$
W^{1} Z\left(I^{n}\right) \hookrightarrow X^{\mathrm{op}}\left(I^{n}\right) .
$$

In our setting, we recall that in Theorem 4.4 we have studied an analogous problem in the context of mixed norm spaces. More precisely, we have found the smallest space of the form $\mathcal{R}\left(X, L^{\infty}\right)$, namely $\mathcal{R}\left(X_{W^{1} Z, L^{\infty}}, L^{\infty}\right)$, that verifies

$$
W^{1} Z\left(I^{n}\right) \hookrightarrow \mathcal{R}\left(X_{W^{1} Z, L^{\infty}}, L^{\infty}\right) .
$$

Now, our goal is to compare the optimal r.i. range space with the optimal mixed norm space. We will show in Theorem 5.3 that the following chain of embeddings holds:

$$
W^{1} Z\left(I^{n}\right) \hookrightarrow \mathcal{R}\left(X_{W^{1} Z, L^{\infty}}, L^{\infty}\right) \hookrightarrow X^{\mathrm{op}}\left(I^{n}\right) .
$$

To this end, we first need to recall the following result [23].

Theorem 5.1. Let $Y\left(I^{n}\right)$ and $Z\left(I^{n}\right)$ be r.i. spaces. Then, the Sobolev embedding

$$
W^{1} Z\left(I^{n}\right) \hookrightarrow Y\left(I^{n}\right)
$$

holds if and only if

$$
\left\|\int_{t}^{1} t^{1 / n-1} f(t) d t\right\|_{\bar{Y}(0,1)} \lesssim\|f\|_{\bar{Z}(0,1)}, \quad f \in \bar{Z}(0,1) .
$$


Remark 5.2. We would also like to emphasize that Theorem 5.1 was used in 23 . to prove Sobolev estimates as well as to give the following characterization of the optimal range space when the domain space is given:

$$
\left(X^{\mathrm{op}}\right)^{\prime}\left(I^{n}\right)=\left\{f \in \mathcal{M}\left(I^{n}\right):\|f\|_{\left(X^{\mathrm{op}}\right)^{\prime}\left(I^{n}\right)}=\left\|t^{1 / n} f^{* *}(t)\right\|_{\bar{Z}^{\prime}(0,1)}<\infty\right\} .
$$

As a consequence, for instance, they recovered the classical estimates by Poornima [31], O'Neil [29] and Peetre [30]

$$
W^{1} L^{p}\left(I^{n}\right) \hookrightarrow L^{n p /(n-1 p), p}\left(I^{n}\right),
$$

and the so-called limiting or critical case of Sobolev embedding due to Hansson [21], Brezis and Wainger [12] and Maz'ya [26]

$$
W^{1} L^{n}\left(I^{n}\right) \hookrightarrow L^{\infty, n ;-1}\left(I^{n}\right) .
$$

Furthermore, as a new contribution, the authors showed that the range spaces $L^{n p /(n-p), p}\left(I^{n}\right)$ and $L^{\infty, n ;-1}\left(I^{n}\right)$ in (41) and (42) respectively, are the best possible among r.i. spaces. We now see that we can further improve these results.

Theorem 5.3. Let $Z\left(I^{n}\right)$ be an r.i. space, let $X^{\mathrm{op}}\left(I^{n}\right)$ be the optimal r.i. space in (38) and let $\mathcal{R}\left(X_{W^{1} Z, L^{\infty}}, L^{\infty}\right)$ be the smallest space of the form $\mathcal{R}\left(X, L^{\infty}\right)$ that verifies (39). Then,

$$
W^{1} Z\left(I^{n}\right) \hookrightarrow \mathcal{R}\left(X_{W^{1} Z, L^{\infty}}, L^{\infty}\right) \hookrightarrow X^{\mathrm{op}}\left(I^{n}\right) .
$$

Moreover, $X^{\mathrm{op}}\left(I^{n}\right)$ is the smallest r.i. space that verifies

$$
\mathcal{R}\left(X_{W^{1} Z, L^{\infty}}, L^{\infty}\right) \hookrightarrow X^{\mathrm{op}}\left(I^{n}\right) .
$$

Proof. We fix $\mathcal{R}\left(X_{W^{1} Z, L^{\infty}}, L^{\infty}\right)$. Using now Theorem 3.3, we construct the smallest r.i. space, denoted by $Y_{\mathcal{R}\left(X_{\left.W^{1} Z, L^{\infty}, L^{\infty}\right)}\right.}\left(I^{n}\right)$, that verifies

$$
\mathcal{R}\left(X_{W^{1} Z, L^{\infty}}, L^{\infty}\right) \hookrightarrow Y_{\mathcal{R}\left(X_{W^{1} Z, L^{\infty}, L^{\infty}}\right)}\left(I^{n}\right) .
$$

Then, by (39), it follows that

$$
W^{1} Z\left(I^{n}\right) \hookrightarrow Y_{\mathcal{R}\left(X_{W^{1} Z, L^{\infty}}, L^{\infty}\right)}\left(I^{n}\right),
$$

and hence, our assumption on $X^{\mathrm{op}}\left(I^{n}\right)$ implies that

$$
X^{\mathrm{op}}\left(I^{n}\right) \hookrightarrow Y_{\mathcal{R}\left(X_{\left.W^{1} Z, L^{\infty}, L^{\infty}\right)}\left(I^{n}\right) .\right.}
$$

On the other hand, if $f \in X^{\mathrm{op}}\left(I^{n}\right)$, then, using a change of variables, we get

$$
\begin{aligned}
\|f\|_{X^{\mathrm{op}}\left(I^{n}\right)} & =\sup _{\|g\|_{\left(X^{\mathrm{op})^{\prime}\left(I^{n}\right)} \leq 1\right.}} \int_{0}^{1} f^{*}(t) g^{*}(t) d t \\
& \leq \sup _{\|g\|_{\left(X^{\mathrm{op})^{\prime}\left(I^{n}\right)} \leq 1\right.}} \int_{0}^{1} f^{*}(t) t^{-1 / n} \sup _{t<s<1} s^{1 / n} g^{*}(s) d t \\
& \approx \sup _{\|g\|_{\left(X^{\mathrm{op})^{\prime}\left(I^{n}\right)} \leq 1\right.}} \int_{0}^{1} f^{*}\left(t^{n^{\prime}}\right) \sup _{t^{n^{\prime}<s<1}} s^{1 / n} g^{*}(s) d t
\end{aligned}
$$

and hence, by Hölder's inequality, we obtain that

$$
\|f\|_{X^{\mathrm{op}\left(I^{n}\right)}} \lesssim \sup _{\|g\|_{\left(X^{\mathrm{op}}\right)^{\prime}\left(I^{n}\right)} \leq 1}\left\|f^{*}\left(t^{n^{\prime}}\right)\right\|_{\bar{X}_{W^{1} Z, L^{\infty}}(0,1)}\left\|_{\sup _{t^{n^{\prime}<s<1}} s^{1 / n} g^{*}(s)}\right\|_{\bar{X}_{W^{1} Z, L^{\infty}}^{\prime}(0,1)} .
$$


But, combining Theorem 4.4 and (40), we have that

$$
\begin{aligned}
\left\|\sup _{t^{n^{\prime}<s<1}} s^{1 / n} g^{*}(s)\right\|_{\bar{X}_{W^{1} Z, L}^{\prime}(0,1)} & \approx\left\|t^{-1 / n^{\prime}} \int_{0}^{t} y^{-1 / n} \sup _{y<s<1} s^{1 / n} g^{*}(s) d y\right\|_{\bar{Z}^{\prime}(0,1)} \\
& =\left\|t^{-1 / n} \sup _{t<s<1} s^{1 / n} g^{*}(s)\right\|_{{\overline{X^{\mathrm{op}}}}^{\prime}(0,1)},
\end{aligned}
$$

and so, by [23, Remark 3.11], we deduce that

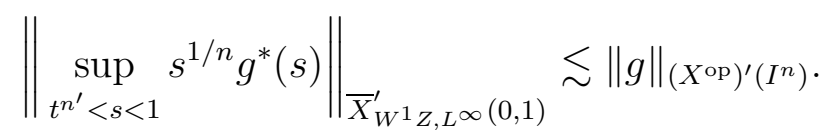

Therefore, using this fact and (45), we get

$$
\|f\|_{X^{\mathrm{op}}\left(I^{n}\right)} \lesssim\left\|f^{*}\left(t^{n^{\prime}}\right)\right\|_{\bar{X}_{W^{1} Z, L}(0,1)} .
$$

and hence, by Theorem 3.3, we conclude that

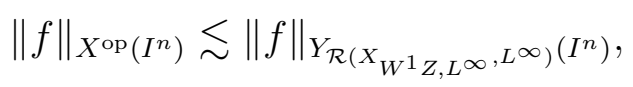

from which it follows that

$$
Y_{\mathcal{R}\left(X_{W^{1} Z, L^{\infty}}, L^{\infty}\right)}\left(I^{n}\right) \hookrightarrow X^{\mathrm{op}}\left(I^{n}\right) .
$$

As a consequence, combining (44) and (46) yields

$$
X^{\mathrm{op}}\left(I^{n}\right)=Y_{\mathcal{R}\left(X_{W^{1} Z, L^{\infty}, L^{\infty}}\right)}\left(I^{n}\right),
$$

and so, (39) and (43) imply that

$$
W^{1} Z\left(I^{n}\right) \hookrightarrow \mathcal{R}\left(X_{W^{1} Z, L^{\infty}}, L^{\infty}\right) \hookrightarrow X^{\mathrm{op}}\left(I^{n}\right)=Y_{\mathcal{R}\left(X_{W^{1} Z, L^{\infty}, L^{\infty}}\right)}\left(I^{n}\right),
$$

as we wanted to show.

As a consequence of Theorem 5.3, we shall see that the classical estimates for the standard Sobolev space $W^{1} L^{p}\left(I^{n}\right)$ by Poornima [31, O'Neil [29] and Peetre [30] $(1 \leq p<n)$, and by Hansson [21] and Brezis and Wainger [12] and Maz'ya [26] $(p=n)$ can be improved considering mixed norms on the target spaces.

Corollary 5.4. Let $1 \leq p<n$. Then,

$$
W^{1} L^{p}\left(I^{n}\right) \hookrightarrow \mathcal{R}\left(L^{p(n-1) /(n-p), p}, L^{\infty}\right) \underset{\neq}{\hookrightarrow} L^{p n /(n-p), p}\left(I^{n}\right) .
$$

Proof. If $Z\left(I^{n}\right)=L^{p}\left(I^{n}\right)$, with $1 \leq p<n$, then, by Remark 5.2 and Corollary 4.5, we have

$$
X^{\mathrm{op}}\left(I^{n}\right)=L^{p n /(n-p), p}\left(I^{n}\right) \text { and } \mathcal{R}\left(X_{W^{1} Z, L^{\infty}}, L^{\infty}\right)=\mathcal{R}\left(L^{p(n-1) /(n-p), p}, L^{\infty}\right) .
$$

Therefore, using Theorem 5.3 and Theorem 3.4 , the result follows immediately.

Corollary 5.5. $W^{1} L^{n}\left(I^{n}\right) \hookrightarrow \mathcal{R}\left(L^{\infty, n ;-1}, L^{\infty}\right) \underset{\neq}{\hookrightarrow} L^{\infty, n ;-1}\left(I^{n}\right)$.

Proof. Use Corollary 4.6 instead of Corollary 4.5 and argue as in the proof of Corollary [5.4.

Acknowledgments: We would like to thank the referee for his/her careful revision which has improved the final version of this work. 


\section{REFERENCES}

[1] R. A. Adams and J. J. F. Fournier, Sobolev Spaces, second ed., Pure and Applied Mathematics (Amsterdam), vol. 140, Elsevier/Academic Press, Amsterdam, 2003.

[2] R. Algervik and V. I. Kolyada, On Fournier-Gagliardo mixed norm spaces, Ann. Acad. Sci. Fenn. Math. 36 (2011), no. 2, 493-508.

[3] S. Barza, A. Kamińska, L. Persson, and J. Soria, Mixed norm and multidimensional Lorentz spaces, Positivity 10 (2006), no. 3, 539-554.

[4] A. Benedek and R. Panzone, The space $L^{p}$, with mixed norm, Duke Math. J. 28 (1961), 301-324.

[5] C. Bennett and K. Rudnick, On Lorentz-Zygmund spaces, Dissertationes Math. 175 (1980), $1-72$.

[6] C. Bennett and R. Sharpley, Interpolation of Operators, Pure and Applied Mathematics, vol. 129, Academic Press Inc., Boston, MA, 1988.

[7] J. Bergh and J. Löfström, Interpolation Spaces. An Introduction, Springer-Verlag, Berlin, 1976.

[8] R. C. Blei and J. J. F. Fournier, Mixed-norm conditions and Lorentz norms, Commutative harmonic analysis (Canton, NY, 1987), vol. 91, 1989, pp. 57-78.

[9] A. P. Blozinski, Multivariate rearrangements and Banach function spaces with mixed norms, Trans. Amer. Math. Soc. 263 (1981), 149-167.

[10] A. Boccuto, A. V. Bukhvalov, and A. R. Sambucini, Some inequalities in classical spaces with mixed norms, Positivity 6 (2002), 393-411.

[11] H. Brezis, Analyse Fonctionnelle, Collection Mathématiques Appliqués pour la Maîtrise, Masson, Paris, 1983.

[12] H. Brezis and S. Wainger, A note on limiting cases of Sobolev embeddings and convolution inequalities, Comm. Partial Differential Equations 5 (1980), no. 7, 773-789.

[13] A. V. Buhvalov, Spaces with mixed norm, Vestnik Leningrad. Univ. (1973), no. 19 Mat. Meh. Astronom. Vyp. 4, 5-12, 151.

[14] M. J. Carro, L. Pick, J. Soria, and V. D. Stepanov, On embeddings between classical Lorentz spaces, Math. Inequal. Appl. 4 (2001), 397-428.

[15] A. Cianchi, Symmetrization and second-order Sobolev inequalities, Ann. Mat. Pura Appl. (4) 183 (2004), no. 1, 45-77.

[16] N. Clavero and J. Soria, Mixed norm spaces and rearrangement invariant estimates, J. Math. Anal. Appl. 419 (2014), 878-903.

[17] R. DeVore and K. Scherer, Interpolation of linear operators on Sobolev spaces, Ann. of Math. 109 (1979), 583-599.

[18] D. E. Edmunds, R. Kerman, and L. Pick, Optimal Sobolev imbeddings involving rearrangementinvariant quasinorms, J. Funct. Anal. 170 (2000), 307-355.

[19] J. J. F. Fournier, Mixed norms and rearrangements: Sobolev's inequality and Littlewood's inequality, Ann. Mat. Pura Appl. 148 (1987), 51-76.

[20] E. Gagliardo, Proprietà di alcune classi di funzioni in più variabili, Ricerche Mat. 7 (1958), $102-137$.

[21] K. Hansson, Imbedding theorems of Sobolev type in potential theory, Math. Scand. 45 (1979), no. $1,77-102$.

[22] T. Holmstedt, Interpolation of quasi-normed spaces, Math. Scand. 26 (1970), 177-199.

[23] R. Kerman and L. Pick, Optimal Sobolev imbeddings, Forum Math. 18 (2006), no. 4, 535-570.

[24] V. I. Kolyada, Iterated rearrangements and Gagliardo-Sobolev type inequalities, J. Math. Anal. Appl. 387 (2012), 335-348.

[25] _ On Fubini type property in Lorentz spaces, Recent Advances in Harmonic Analysis and Applications 25 (2013), 171-179.

[26] V. Maz'ya, Sobolev Spaces, Springer Series in Soviet Mathematics, Springer-Verlag, Berlin, 1985.

[27] M. Milman, Notes on interpolation of mixed norm spaces and applications, Quart. J. Math. Oxford Ser. (2) 42 (1991), no. 167, 325-334.

[28] L. Nirenberg, On elliptic partial differential equations, Ann. Scuola Norm. Sup. Pisa 13 (1959), 115-162. 
[29] R. O'Neil, Convolution operators and L(p, q) spaces, Duke Math. J. 30 (1963), 129-142.

[30] J. Peetre, Espaces d'interpolation et théorème de Soboleff, Ann. Inst. Fourier (Grenoble) 16 (1966), no. fasc. 1, 279-317.

[31] S. Poornima, An embedding theorem for the Sobolev space $W^{1,1}$, Bull. Sci. Math. (2) 107 (1983), no. 3, 253-259.

[32] S. L. Sobolev, On a theorem of functional analysis, Math. Sb. 46 (1938), 471-496, translated in Amer. Math. Soc. Transl. 34 (1963), 39-68.

[33] G. Talenti, Inequalities in rearrangement invariant function spaces, Nonlinear analysis, function spaces and applications, Vol. 5 (Prague, 1994), Prometheus, Prague, 1994, pp. 177-230.

Department of Applied Mathematics and Analysis, University of Barcelona, Gran Via 585, E-08007 Barcelona, Spain

E-mail address: nadiaclavero@ub.edu

Department of Applied Mathematics and Analysis, University of Barcelona, Gran Via 585, E-08007 Barcelona, Spain

E-mail address: soria@ub.edu 